\title{
Heat transfer in nanofluid spray cooling of a solid heated surface for cooling systems in civil and military applications
}

\author{
M. Sanches ${ }^{* 1}$, A. S. Moita ${ }^{2,1 *}$ A. P. C. Ribeiro ${ }^{3}$, A.L.N. Moreira ${ }^{1}$, \\ ${ }^{1} \mathrm{IN}+$ Instituto Superior Técnico, Universidade de Lisboa, Lisbon, Portugal \\ ${ }^{2}$ CINAMIL, Dep. Exact Sciences and Engineering, Academia Militar, Amadora, Portugal \\ ${ }^{3}$ CQE Instituto Superior Técnico, Universidade de Lisboa, Lisbon, Portugal \\ *Corresponding author email: anamoita@tecnico.ulisboa.pt, moita.asoh@exercito.pt
}

\begin{abstract}
Interest in spray cooling has been increasing as an efficient thermal management technique for high power load systems such as electronics, e.g. in UAV's (Unmanned Aerial Vehicles), which have an important application in both civil and military applications. This work aimed to study the use of nanofluids as a way of improving sprays thermal performance. Nanofluids were prepared using, as base solution, distilled water with $0.05 \%(\mathrm{~m} / \mathrm{m})$ cetyltrimethylammonium bromide. Alumina nanoparticles were mixed at different concentrations: $0.5 \%, 1 \%$ and $2 \%(\mathrm{~m} / \mathrm{m})$. The effect of the shape of the nanoparticles was inferred using two different silver nanofluids, with $1 \%(\mathrm{~m} / \mathrm{m})$ concentration, one using spherical and the other using triangular particles. These nanofluids were sprayed using a hollow-cone atomizer, at two different heights from an AISI 304 stainless steel foil, heated by Joule effect. Thermal footprints of the sprays were acquired using infrared thermography and dissipated heat fluxes were calculated. When comparing the performance of silver nanofluids, the one with spherical particles resulted in slightly lower surface temperatures than the fluid with triangular particles. Heat transfer coefficients decreased with increasing thermal conductivity and dynamic viscosity of the nanofluids. On the other hand, a strong positive correlation was revealed with the specific heat capacity of the nanofluids. For the range of experimental conditions covered here, nanofluids have proven to increase the thermal performance of the spray.
\end{abstract}

\section{Keywords}

Spray cooling, Nanofluids, Infrared thermography, Heat transfer.

\section{Introduction}

The dissipation of high thermal loads is still an obstacle to overcome in several applications such as in electronics cooling, UAV's (for military applications), among others [1,2]. Indeed, defense and military systems are at the top of the most demanding applications, as they require simple but robust solutions, which endure under extremely harsh environmental conditions. They also require low maintenance effort and costs. However, as technology evolves, there is a significant attempt in developing defense systems which are growingly more sustainable, trying to decrease its environmental impact. In this context, UAV's Unmanned Aerial Vehicles have gained significant relevance in defense applications. Primarily used to conduct intelligence, surveillance and reconnaissance activities, military UAVs are now being developed for a wide range of uses such as electronic attack (EA), strike missions, suppression/destruction of enemy air defense (SEAD), network nodes, communication relays, among others, being the key demand generator for drones in 2018 in the US. [3] Such small and complex electronic systems often require for effective thermal management, for which spray cooling offers a high potential solution [2]. However, further improvements are still addressed, for instance towards the use of nanofluids. Nanofluids are 
composed of a basic fluid to which nanometer particles (of sizes up to 100nm) of several materials such as metals (and metal oxides) are added [4]. Nanofluids have been extensively used in several flow geometries [5-6], but are still not much explored in spray cooling systems. In spray cooling applications, several authors report the difficulty in getting benefits from the surfactants addition to the fluids, to improve their stability, without disfavoring other fundamental physical fluid properties, as the bulk surface tension and the surface wettability that affect the nebulization process and the spray wall interaction event [7-9]. Also, many studies on spray cooling characterization still rely on the use of temperature sensors, such as thermocouples, to estimate the dissipated heat flux [10]. Time resolved thermography can provide details on the heat transfer at spray-surface interactions, but there are still several issues, which preclude its wide use to characterize the heat transfer mechanisms observed in spray cooling.

In this context, the present work addresses, in a systematic approach, the effect of the surfactant properties in the spray dynamics and consequently on the heat transfer mechanisms at spray cooling on a solid metallic and smooth surface. This work further details, making use of an advance post-processing procedure of thermal images obtained by time resolved infrared thermography, the effect of the concentration, nature (material) and shape of the nanoparticles. The main conclusions are then discussed at the light of practical applications on electronics cooling.

\section{Material and Methods \\ Experimental set-up}

Different nanofluids, composed by alumina and silver nanoparticles (with mass concentrations ranging between $0.5 \%$ and $2 \% \mathrm{~m} / \mathrm{m}$ ) combined with a base fluid of distilled water with $0.05 \%$ $(\mathrm{m} / \mathrm{m})$ cetyltrimethylammonium bromide - CTAB, were atomized using a tangential pressureswirl atomizer that creates a hollow cone spray. The atomizer, which was used in previous works [9], has a discharge orifice of $0.42 \mathrm{~mm}$ in diameter and two opposing tangential inlet ports with a squared shape cross section of $0.6 \times 0.6 \mathrm{~mm}^{2}$. It operates at an ejection pressure of $0.5 \mathrm{MPa}$, delivering a mass flow rate of $7 \mathrm{~kg} / \mathrm{h}$. The resulting hollow-cone spray was characterized in terms of droplet size and velocity distributions using Phase Doppler Anemometry by Malý et al. [11] and by Figueiredo et al. [9].

The spray impacts on a stainless-steel foil AISI 304, with a width of $60 \mathrm{~mm}$, length of $90 \mathrm{~mm}$ and thickness of $20 \mu \mathrm{m}$, which was heated by Joule effect, being the power provided by a HP 6274B DC power supply. The surface was characterized in terms of surface topography and wettability. Surface topography was characterized using a profilemeter (a Dektak 3 from Veeco), with a vertical resolution of $20 \mathrm{~nm}$. The surface is perfectly smooth within this vertical resolution. The wettability was characterized based on the measurement of the equilibrium contact angle, using an optical tensiometer (THETA from Attention), based on the sessile droplet method, as detailed for instance in [9]. The static contact angle was $\theta=86.5 \pm 6.4^{\circ}$ for water on the AISI304 stainless steel surface, measured at $20^{\circ} \mathrm{C} \pm 3^{\circ} \mathrm{C}$. As the surfactant is added, the contact angle decreases, as expected, to $\theta=53.6 \pm 6.3^{\circ}$. Then, the contact angle slightly decreases between $61.8 \pm 2.9^{\circ}<\theta<57.9 \pm 11.3^{\circ}$ for the alumina nanofluids, as the alumina concentration increases from $0.5 \mathrm{wt} \%$ to $2 \%$. Regarding the silver nanofluids, $\theta$ $=51.1 \pm 3.5^{\circ}$ for the silver nanofluid with the spherical particles and $\theta=47.3 \pm 6.3^{\circ}$ for the silver nanofluid with the triangular particles. 


\section{Preparation and characterization of the nanofluids}

Nanofluid synthesis followed the procedure described in our previous study [9]. The nanofluids were prepared at the Structural Chemistry Center of the Department of Chemical Engineering at Instituto Superior Técnico of Lisbon. The study addressed the effect of the concentration, material and shape of the nanoparticles. The effect of the concentration was focused on the alumina nanofluids, in which the nanoparticles concentration was varied between $0.5 w t \%$ and $2 w t \%$. Alumina and silver nanofluids were compared for the same nanoparticles concentration (1wt\%) to infer on the effect of the nanoparticles material. Finally, spherical and triangular silver nanoparticles (1wt\%) were used to investigate the effect of the shape of the nanoparticles. Shape and size of the nanoparticles were inferred by SEM - Scanning Electron Microscope analysis. All the nanoparticles were dispersed in the same base fluid composed of distilled water and $0.05 \mathrm{wt} \%$ CTAB. The fluids were characterized in terms of specific mass

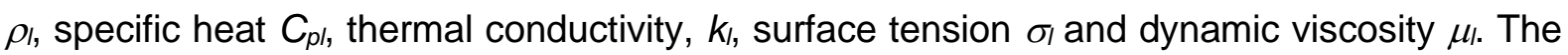
specific mass, $\rho_{l}$ of the nanofluids fluids was calculated as reported by Sharma et al. [12]. The specific heat capacity $C_{p l}$ was determined as in Qi et al. [13]. The dynamic viscosity, on the other hand, was experimentally measured at $20^{\circ} \mathrm{C}$, using a TA instruments ARI 500 ex rheometer. The measurements have an accuracy of $\pm 5 \%$. Finally, the surface tension was measured using the optical tensiometer THETA, from Attention. 15 measurements were performed for each fluid, at $20^{\circ} \mathrm{C} \pm 3^{\circ} \mathrm{C}$, using the pendant drop method. Detailed description of the procedure followed can be described in [9].

Table 1 summarizes the thermophysical properties of all the fluids studied in the present work.

Table 1. Thermophysical properties of the working fluids.

\begin{tabular}{|c|c|c|c|c|c|}
\hline Fluid composition & $\begin{array}{l}\rho_{l} \\
{\left[\mathrm{~kg} / \mathrm{m}^{3}\right]}\end{array}$ & $\begin{array}{l}C_{p l} \\
{[\mathrm{~kJ} /(\mathrm{kg} . \mathrm{K})]}\end{array}$ & $k_{l}[\mathrm{~W} /(\mathrm{m} \cdot \mathrm{K})]$ & $\sigma_{l}[\mathrm{mN} / \mathrm{m}]$ & $\begin{array}{l}\mu_{l} \\
\text { [mPa.s] }\end{array}$ \\
\hline Water & 998 & 4.22 & 0.6060 & $74.6 \pm 2.2$ & 1.009 \\
\hline Water+0.05\%(m/m) CTAB & 999 & - & - & $35.9 \pm 0.9$ & 1.291 \\
\hline $\begin{array}{l}\text { Water }+0.05 \%(\mathrm{~m} / \mathrm{m}) \\
\text { CTAB }+0.5 \%(\mathrm{~m} / \mathrm{m}) \\
\text { Alumina }\end{array}$ & 1002 & 4.20 & 0.6082 & $39.0 \pm 1.3$ & 3.572 \\
\hline $\begin{array}{l}\text { Water }+0.05 \%(\mathrm{~m} / \mathrm{m}) \\
\text { CTAB }+1 \%(\mathrm{~m} / \mathrm{m}) \text { Alumina }\end{array}$ & 1006 & 4.19 & 0.6104 & $40.2 \pm 2.7$ & 6.815 \\
\hline $\begin{array}{l}\text { Water }+0.05 \%(\mathrm{~m} / \mathrm{m}) \\
\text { CTAB }+2 \%(\mathrm{~m} / \mathrm{m}) \text { Alumina }\end{array}$ & 1011 & 4.15 & 0.6149 & $40.2 \pm 0.9$ & 8.835 \\
\hline $\begin{array}{l}\text { Water }+0.05 \%(\mathrm{~m} / \mathrm{m}) \\
\text { CTAB }+1 \%(\mathrm{~m} / \mathrm{m}) \quad \text { Silver } \\
\text { (Spherical) }\end{array}$ & 1023 & 4.12 & 0.6106 & $40.0 \pm 0.4$ & 13.175 \\
\hline $\begin{array}{l}\text { Water }+0.05 \%(\mathrm{~m} / \mathrm{m}) \\
\text { CTAB }+1 \%(\mathrm{~m} / \mathrm{m}) \quad \text { Silver } \\
\text { (Triangular) }\end{array}$ & 1023 & 4.12 & 0.6096 & $43.1 \pm 0.4$ & 18.903 \\
\hline
\end{tabular}

\section{Measurement procedures}

After measuring the temperature of the fluid to be tested, the fluid was placed inside the highpressure vessel. The nozzle height relative to the foil was adjusted, as necessary. Then, the compressed air valve was opened to pressurize the vessel and a thermographic video with 5 frames of the foil before heating is recorded. As for the thermographic images, the best configuration, which resulted in a reasonable recording rate and allowed to capture at least 
$12 \mathrm{~s}$ of the steady-state regime, was found to be a recording rate of 40fps and a length of 1000 frames. Thereafter, the power source was turned on and set to the desired current. While the foil temperature increased, the ambient temperature and the difference of electric potential between the contacts were checked using a multimeter and registered. Once the temperature of the foil has stabilized, the thermographic and high-speed recordings were initiated. The solenoid valve was only opened once the thermographic camera recording rate has stabilized. When the recordings have finished, the surface was cleaned using acetone and distilled water. For each experimental condition, three identical tests were performed in a row. When changing the test fluid, all wet parts were cleaned with distilled water and dried.

As aforementioned, the foil was heated by Joule effect, imposing two currents, $(I=10 \mathrm{~A}$ and $I$ $=15 \mathrm{~A}$ ) which correspond to the imposed heat fluxes $q_{\text {soule }}=914.5 \mathrm{~W}$ (and an initial temperature of $T_{0}=84.14^{\circ} \mathrm{C}$ ) and $q_{\text {Joule }}=2104.98 \mathrm{~W}$ (and an initial temperature of $T_{0}=140.97^{\circ} \mathrm{C}$ ). The impact was studied for two distances from the nozzle to the surface of $Z=10 \mathrm{~mm}$ and $Z=20 \mathrm{~mm}$.

The thermal footprints of the sprays impacting on the stainless-steel foil, acquired by the thermographic camera were processed using an adapted version of the thermographic camera video processing MATLAB code developed by Pontes [14], which also describe in detail the calibration and post-processing procedures. The thermographic camera captures the energy emitted by the object and transforms it to a value of Analog to Digital Unit (ADU). Then, the ADU values are converted back to energy intensities by applying the calibration. The ambient temperature is input to the program to subtract the contribution of atmosphere radiation. Thereafter, Stefan-Boltzmann law is applied to obtain object temperature. Finally, a weighted background removal filter and a Gaussian filter with standard deviation 2 and a 9x9 pixel kernel are applied.

The analysis performed here considered a constant temperature across the foil, which is valid for a Biot number $B i=h \delta / k_{s} \ll 1$ [15], where $h$ is the heat transfer coefficient, $\delta$ is the foil thickness and $k_{s}$ is the thermal conductivity of the foil. Such condition is met in the present study since $\delta=O\left(10^{-5}\right) \mathrm{m}, k_{s}=O(101) \mathrm{W} /(\mathrm{m} . \mathrm{K})$ and $h_{l}=O\left(10^{3}\right) \mathrm{W}\left(\mathrm{m}^{2} . \mathrm{K}\right)$. The thermographic videos were used to calculate the dissipated heat flux from the foil, performing pixel energy balances. Main uncertainties are associated to the $K$ thermocouple readings $\left( \pm 0.03^{\circ} \mathrm{C}\right)$, to the analogue pressure gauge ( $\pm 1 \mathrm{psi})$, to the FLUKE 123 SCOPEMETER multimeter $( \pm 1 \mathrm{mv})$ and the current gauge of the HP 6274B DC power supply $( \pm 0.5 \mathrm{~A})$. The atomizer position adjustment knobs have an associated uncertainty of $\pm 0.5 \mathrm{~mm}$.

\section{Results and Discussion}

\section{Heat transfer mechanisms on spray impingement}

Water spray is first taken as a reference to characterize the hydrodynamic and thermal behaviour of the spray. The spray impact can be characterized by two main regimes: the transient regime, in which the surface temperature drastically decreases, from the initial foil temperature to values close to the fluid temperature; and the steady-state regime, where temperatures no longer change with time. Comparing the curves for $Z=10 \mathrm{~mm}$ and $Z=20$ $\mathrm{mm}$ (which are not shown here due to paper length constrains), the foil temperature decreases and stabilizes slightly faster for $Z=20 \mathrm{~mm}$ than for $Z=10 \mathrm{~mm}$. This trend is related to the fact that, by placing the atomizer higher relative to the foil, the spray footprint is larger, thus covering a larger area of the foil, resulting in faster cooling. Regarding the temporal evolution of the dissipated heat flux $q^{\prime \prime}$ diss, for distilled water sprays and working conditions with $I=10 \mathrm{~A}$ and $I=15 \mathrm{~A}$, respectively, the results show that the transient regime is characterized by a spike in the dissipated heat flux, which then decreases. This regime is followed by the steady- 
state regime where, again no variations in the dissipated heat flux are verified. The maximum dissipated heat flux is higher for $Z=20 \mathrm{~mm}$, when compared to that obtained for $Z=10 \mathrm{~mm}$.

\section{Effect of adding the surfactant CTAB}

The values of the heat flux q" diss and heat transfer coefficient $h$ in the different working conditions are underlined for water and base fluid respectively in Figures $1 \mathrm{a}$ and $1 \mathrm{~b}$. The plots show that adding CTAB leads to the increase of the dissipated heat flux, of about $1.3 \%$ for $Z$ $=10 \mathrm{~mm}$ and of $0.35 \%$ for $Z=20 \mathrm{~mm}$, respectively. Furthermore, when comparing the relative mean temperature $\triangle T$, it increases with $C T A B$ for all situations, except for the maximum working conditions $(I=15 \mathrm{~A}$ and $Z=20 \mathrm{~mm}$ ). The maximum increase for the heat flux and heat transfer coefficient occurs when $I=15 \mathrm{~A}$ and $Z=10 \mathrm{~mm}$ and is of approximately $6.9 \%$. Finally, due to the temperature difference, the heat transfer coefficient decreases with the addition of CTAB, except for the maximum working conditions, where an increase of $2.7 \%$ is verified (Figure1b). The maximum decrease occurs for $I=15 \mathrm{~A}$ and $Z=10 \mathrm{~mm}$ and is of approximately $5.1 \%$. The observed lower heat transfer coefficients which are related to the higher temperature profiles, obtained with the addition of CTAB may be related with the increased wettability, which enhances the flow of fluid over the foil. Therefore, the liquid film is less thick, which reduces the thermal performance.

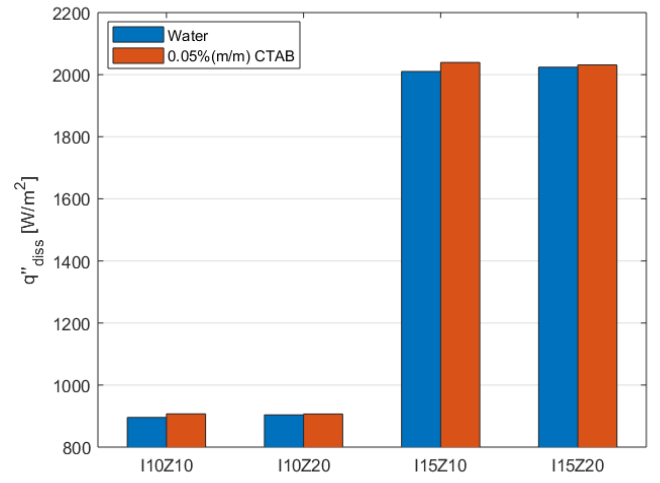

a)

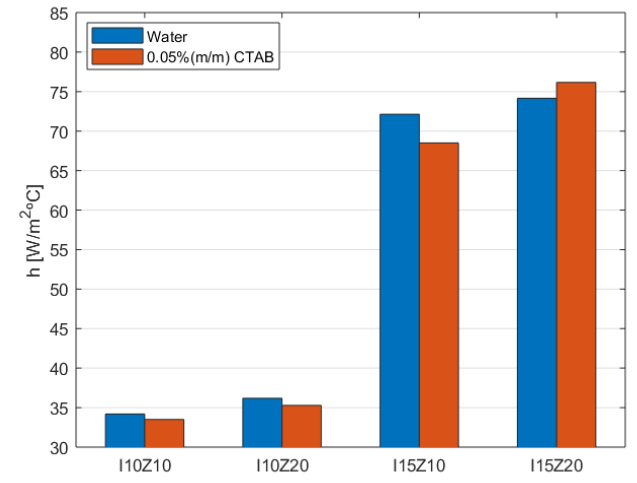

b)

Figure 1. (a) Dissipated heat flux and (b) heat transfer coefficients for water and base fluid sprays impacting on the smooth and heated stainless steel foil, for the different working conditions. In the plots, I10Z10 stands for an imposed current on the foil of $10 \mathrm{~A}$ and an impact height of $Z=10 \mathrm{~mm}$. $110 Z 20$ represents the condition of $10 \mathrm{~A}$ of imposed current and impact height of $Z=20 \mathrm{~mm}$. $/ 15 Z 10$ stands for an imposed current on the foil of $15 \mathrm{~A}$ and an impact height of $Z=10 \mathrm{~mm}$. $/ 15 Z 20$ stands for an imposed current on the foil of $15 \mathrm{~A}$ and an impact height of $Z=$ $20 \mathrm{~mm}$.

\section{Effect of nanoparticles concentration (alumina nanofluids)}

To explore the effect of nanoparticles concentration, the base fluid (water $+0.05 w t \%$ CTAB) is compared to the alumina nanofluids, for concentrations ranging between $[0.5,1,2] \%(\mathrm{~m} / \mathrm{m})$. The dissipated heat flux and heat transfer coefficients for the alumina nanofluids are plotted for the different working conditions respectively in Figures $2 a$ and $2 b$. 


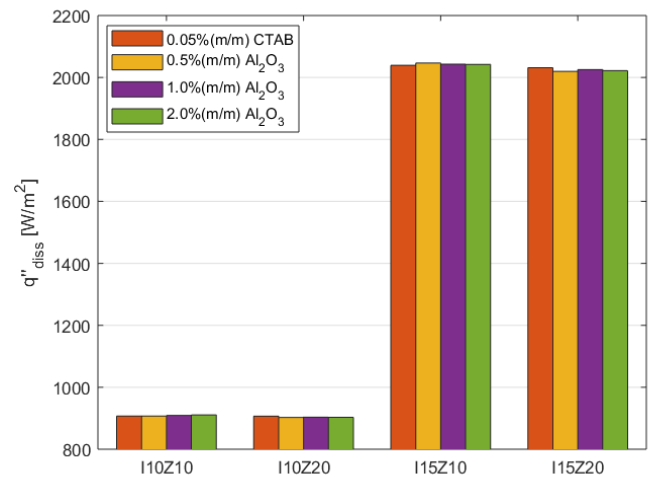

a)

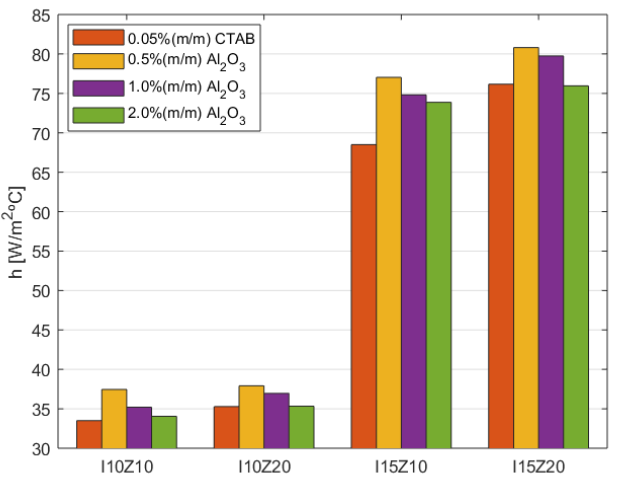

b)

Figure 2. (a) Dissipated heat flux and (b) heat transfer coefficients for alumina nanofluids impacting on the smooth and heated stainless steel foil. The base fluid is water + CTAB is also used as a reference. In the plots, $110 Z 10$ stands for an imposed current on the foil of $10 \mathrm{~A}$ and an impact height of $Z=10 \mathrm{~mm}$. $/ 10 Z 20$ represents

the condition of $10 \mathrm{~A}$ of imposed current and an impact height of $Z=20 \mathrm{~mm}$. $/ 15 Z 10$ stands for an imposed current on the foil of $15 \mathrm{~A}$ and an impact height of $Z=10 \mathrm{~mm}$. $/ 15 Z 20$ stands for an imposed current on the foil of $15 \mathrm{~A}$ and an impact height of $Z=20 \mathrm{~mm}$.

\section{Overall comparison of the different fluids}

Finally, a global comparison of the behavior of the all the nanofluids considered in this work is performed in the present sub-section. Figures 3 and 4 show the behavior of the heat transfer coefficient, as a function of the fluid properties, respectively, of the specific heat capacity $C_{p}$ and of the thermal conductivity $k_{l}$ for the various nanofluids tested here.

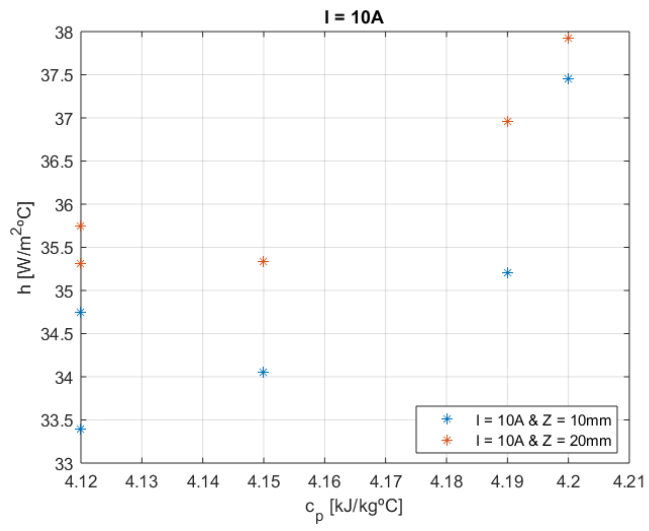

a) $\mathrm{I}=10 \mathrm{~A}$.

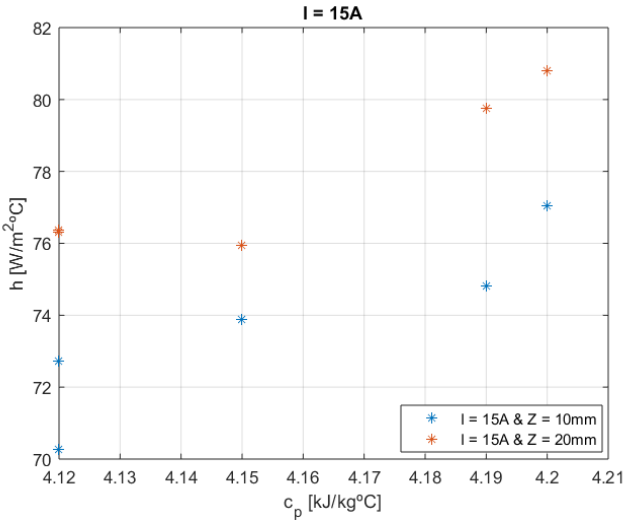

b) $I=15 \mathrm{~A}$.

Figure 3. Heat transfer coefficient as a function of the specific heat capacity of the nanofluids for an imposed current of: $a=10 \mathrm{~A}$ and b) $15 \mathrm{~A}$.
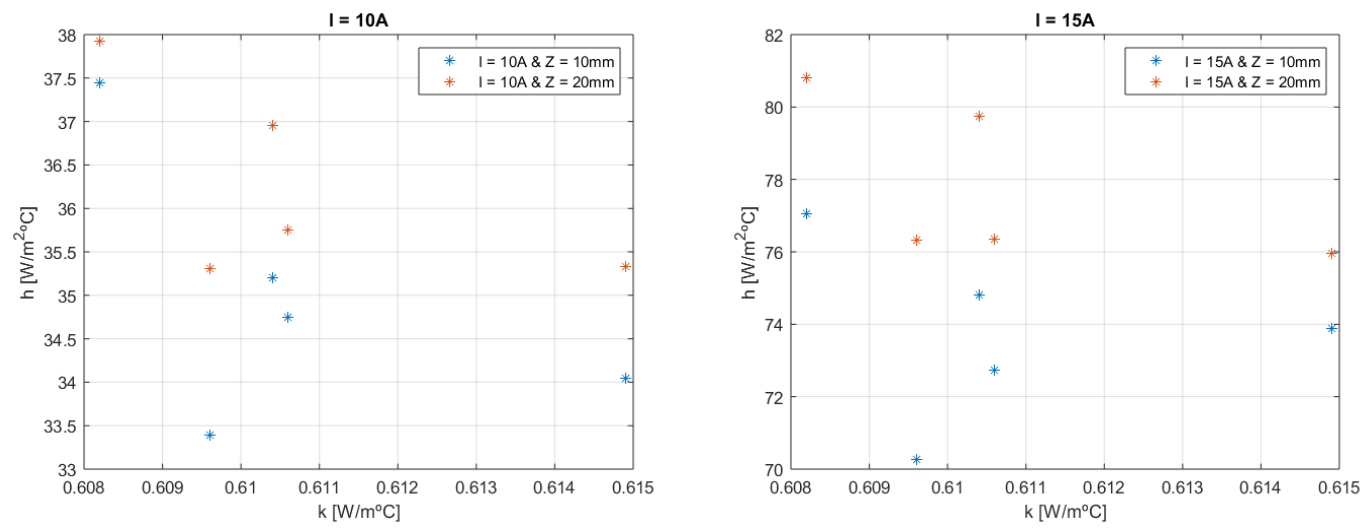

a) $I=10 \mathrm{~A}$.
b) I $=15 \mathrm{~A}$.

Figure 4. Heat transfer coefficient as a function of the thermal conductivity of the nanofluids

Figure 3 marks a positive correlation of the heat transfer coefficient the with specific heat capacity of the fluids. Hence, as the specific heat capacity increases, the heat transfer coefficient tends to increase exponentially. However, when considering the heat transfer coefficient as a function of thermal conductivity of the fluid, these show a negative correlation.

\section{Relevant results for practical applications}

The main trends and conclusions presented so far are mainly relevant, when discussed in the context of practical applicability of spray cooling. As aforementioned in the Introduction, one of the most demanding applications for spray cooling is electronics cooling. The current improvement of actual vehicles is directing many research centers and industries towards an important insertion and scale down of on-board electric systems. This is bringing to important raise of the heat flux to be dissipated to ensure the keeping of the electronic devices in a safe temperature conditions. Considering the need for thermal cooling in UAV's and airspace applications, one of the most demanding applications, particularly when used for military purposes, spray cooling can remove heat fluxes of the order of $102-103 \mathrm{~W} /\left(\mathrm{m}^{2}\right)$, which are required to be dissipated in these applications. Nevertheless, both the entire heat sinks on board and the cooling performance of employed thermal restraint and management are deeply circumscribed. This aspect influences the overall flight apparatus and the vehicle's flight time and range. Considering these thermal issues, the novelty of this work is the purpose of practical utilization of nanofluids to improve the cooling capacity of current heat control methodologies. The results of this research can provide useful information to aerospace and electronics engineers and operators, such as possible performance enhancement modifications to aerospace vehicles in industrial applications.

In this context, the use of nanofluids further enhances the heat transfer coefficients, being a good strategy to further enhance spray cooling performance. The main issue relies on the stability of the nanofluids. Hence, alumina nanofluids are a more stable solution and also more affordable, although particles deposition is still a problem. Hence, the application is valid for short missions. Further work on the fluid stability is worth to be explored in the near future.

\section{Conclusions}

The present work addresses the evaluation of nanoparticles concentration and shape in the thermal properties of the nanofluids and in the consequential potential improve in the heat transfer, for spray cooling applications. The base fluid considered is distilled water with surfactant cetyltrimethylammonium bromide (CTAB). Alumina and silver were the nanoparticles used. Thermal footprints of the spray impacting on a heated, smooth stainlesssteel foil are evaluated to quantify temperature gradients and heat fluxes transferred during spray impingement. The results evidence a mild effect of the nanoparticles shape on the silver nanoparticles: higher heat transfer coefficients are observed for the silver nanofluids using spherical particles, when compared to the silver nanofluids using triangular nanoparticles. Higher nanoparticles concentration overall leads to the increase in the thermal conductivity and dynamic viscosity of the nanofluids but decreases the heat transfer coefficients. On the other hand, nanofluids specific heat capacity is increased which results in an enhanced spray cooling capacity.

\section{Acknowledgments}


Authors are grateful to Fundação para a Ciência e Tecnologia (FCT) for partially financing the research under the framework of the project $\mathrm{n}^{\circ} 030171$ financed by LISBOA-01-0145-FEDER030171 / PTDC/EME-SIS/30171/2017 and of the project JICAM/0003/2017.

\section{References}

[1] Kim, J., Int. J. Heat Fluid Flow, vol. 28, no. 4, pp. 753-767, 2007.

[2] Kinney, D., COTS J. J. Military Electron. Comput2: 5-6, 2009.

[3] Army Technology: Drones in Aerospace and Defence: Macroeconomic Trends (armytechnology.com).

[4] Choi, S. U. S., Eastman JA. Enhancing thermal conductivity of fluids with nano- particles. ASME; 1995.

[5] Mo, S., Chen, Y., Jia, L., Luo, X., Applied Energy; vol. 93, pp. 65-70, 2012.

[6] Mehralia, M., Ghatkesar, M. K, Pecnik, R., Applied Energy, vol. 224, pp. 103-115, 2018.

[7] Mansour, R. B., Galanis, N., Nguyen, C. T., Applied Thermal Eng., vol. 27, pp. 240-249, 2007.

[8] Lyu, Z., Asadi, A., Alarifi, I. M., Ali, V., Foong, L. K., Scientific Reports vol. 10, pp. 5185, 2020

[9] Figueiredo, M., Marseglia, G., Moita, A.S., Panão, M.R.O., Ribeiro, A.P.C., Medaglia, C.M. Moreira, A.L.N., Energies, vol. 13(22), pp. 5864 (2929).

[10] Nianyong, Z., Fujiang, C., Yuchun, C., Mengmeng, C., Yu, W., Applied Thermal Eng., vol. 112, pp. 1117-1128, 2017.

[11] Maly, M., Moita, A.S., Jedelsky, J., Ribeiro, A.P.C., Moreira, A. L. N., J. Thermal Analysis and Calorimetry, vol. 135, pp. 3375-3386, 2019.

[12] Sharma, K. V., Sarma, P. K., Azmi, W. H., Mamat, R., Kadirgama, K., The Int. J. Microscale and Nanoscale Thermal and Fluid Transport, vol. 3(4), 2012.

[13] Qi, W. Q., Wang, M. P., Liu, Q. H., J. Materials Sci., vol. 40, pp. 2737-2739, 2005.

[14] Teodori, E., Pontes, P., Moita, A.S., Moreira, A.L.N., Exp. Thermal Fluid Sci., vo. 96, pp. 284-294, 2018.

[15] Incropera, F. P., DeWitt, D. P., Bergman, T. L., Lavine, A. S., Fundamentals of Heat and Mass Transfer. Wiley, 6th edition, 2007. 